\title{
Optimal cut-off points for waist circumference in the definition of metabolic syndrome in Chile
}

\author{
Fanny Petermann-Rocha ${ }^{1,2, *}$ (1), María Adela Martínez-Sanguinetti ${ }^{3}$, Frederick K Ho ${ }^{1}$ (1), \\ Carlos Celis-Morales ${ }^{1,2,4,5,} \dagger$ (1) and Alonso Pizarro6, $\dagger$ (1) on behalf of ELHOC Research \\ Group \\ 'Institute of Health and Wellbeing, University of Glasgow, Glasgow, UK: ${ }^{2}$ British Heart Foundation Glasgow, \\ Cardiovascular Research Centre, Institute of Cardiovascular and Medical Sciences, University of Glasgow, Glasgow \\ G12 8TA, UK: ${ }^{3}$ Instituto de Farmacia, Facultad de Ciencias, Universidad Austral de Chile, Valdivia, Chile: ${ }^{4}$ Centro de \\ Investigación en Fisiología del Ejercicio (CIFE), Universidad Mayor, Santiago, Chile: ${ }^{5}$ Research Group in Education, \\ Physical Activity and Health (GEEAFyS), Universidad Católica del Maule, Talca, Chile: ${ }^{6}$ Department of European and \\ Mediterranean Cultures: Architecture, Environment and Cultural Heritage (DICEM), University of Basilicata, Matera, Italy
}

Submitted 19 October 2019: Final revision received 31 March 2020: Accepted 16 April 2020: First published online 3 July 2020

\begin{abstract}
Objective: To identify sex-specific cut-off points for waist circumference (WC) in the definition of metabolic syndrome (MetS) for the Chilean adult population. Design: MetS was defined as the presence of at least two out of four of the following criteria: TAG $\geq 1.7 \mathrm{mmol} / \mathrm{l}$; HDL-cholesterol: $<1.3 \mathrm{mmol} / \mathrm{l}$ in women and $<1.0 \mathrm{mmol} / \mathrm{l}$ in men; systolic blood pressure $\geq 130 \mathrm{mmHg}$ and/or diastolic blood pressure $\geq 85 \mathrm{mmHg}$; and fasting glucose $\geq 5.6 \mathrm{mmol} / \mathrm{l}$ or current treatment for diabetes. The receiver operating characteristics curve and the AUC were computed to derive the specificity and sensitivity using bootstrapping (10 000 iterations restricted to have at least between 40 and $60 \%$ of the original population). The optimal cut-off point for the Chilean population was computed by sex.

Setting: A representative sample of the Chilean population aged $\geq 15$ years.

Participants: 8182 participants (60\% women) from the three available Chilean National Health Surveys conducted in 2003, 2009-2010 and 2016-2017.

Results: WC had a good predictive ability for MetS (AUC for men 0.74 (95\% CI 0.72, 0.76); AUC for women 0.71 (95\% CI 0.68, 0.73)). The optimal cut-off points for WC, in the definition of MetS, were $92.3 \mathrm{~cm}(95 \%$ CI 90.5, 94.4) and $87.6 \mathrm{~cm}$ (95\% CI $85 \cdot 8,92 \cdot 1)$ for men and women, respectively.

Conclusions: The mentioned cut-off points should be used for WC in the definition of MetS in Chile. As a result, the current recommendation (WHO/International Diabetes Federation) for WC, in the identification of MetS, is not supported by these findings in a representative sample of the Chilean adult population.
\end{abstract}

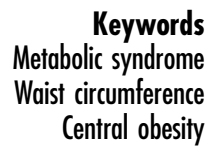

With the increase in the elderly population, globalisation and western lifestyle patterns, obesity and non-communicable diseases, especially cardiovascular disease (CVD), have become a major health burden in the society $^{(1,2)}$. Metabolic syndrome (MetS), defined as a cluster of cardiovascular risk factors, is strongly associated with an increased risk of CVD and premature mortality ${ }^{(3,4)}$.

Reaven was the first to define MetS in 1988, hypothesising that it was a central feature in the development of diabetes and coronary disease ${ }^{(5)}$. Later, the WHO

†C.C.-M. and A.P. contributed equally to this work and are joint senior authors. developed its first formal definition as the combination of insulin resistance, obesity, dyslipidaemia and hypertension $^{(6)}$. Nowadays, this syndrome has been widely recognised as a pathologic condition characterised by the presence of central obesity (as elevated waist circumference (WC)), high blood pressure, high glucose and high blood lipids ${ }^{(7-9)}$. Some organisations have proposed cutoff points for four of its five components: plasma levels of glucose, TAG, low HDL as well as blood pressure ${ }^{(7-9)}$. However, although there is an increasing number of populations for which sex-specific cut-off points have been derived (e.g. Indian, African, Chinese and South Asian

*Corresponding author: Email f.petermann-rocha.1@research.gla.ac.uk

(C) The Author(s), 2020. Published by Cambridge University Press on behalf of The Nutrition Society 
populations) ${ }^{(3)}$, there is not a unique recommendation for central obesity (online Supplementary Table S1). As a result, there is a large number of countries that, due to the lack of available data, are currently using cut-off points derived for white-European populations or other ethnic groups $^{(3)}$.

Epidemiological studies conducted in North America and Europe suggest that some ethnic groups such as South Asian, black and Chinese people experience a higher risk of diabetes at lower levels of obesity than whites ${ }^{(10-13)}$. This suggests that conventional clinical thresholds for obesity that were originally derived from populations of white European background (e.g. the specific case of the body mass index (BMI) $\geq 30 \cdot 0 \mathrm{~kg} / \mathrm{m}^{2}$ or a WC $\geq 88.0 \mathrm{~cm}$ in women or $\geq 102.0 \mathrm{~cm}$ in men) may not be appropriate for non-white groups, such as the Chilean population.

As a consequence, in South American countries where ethnic-specific thresholds for WC have not been proposed, the current recommendation is the use of the already suggested cut-off points for the Asian population $(\geq 90 \cdot 0$ and $\geq 80.0 \mathrm{~cm}$ in men and women, respectively $\left.{ }^{(14)}\right)$. This guidance has been proposed by the WHO and the International Diabetes Federation (IDF) until country-specific information is available(3).

In Chile, the first adopted and used cut-off points were those recommended by the National Cholesterol Education Program Adult Treatment Panel III (ATP III) derived for the US population $(\geq 102.0$ and $\geq 88.0 \mathrm{~cm}$ in men and women, respectively) ${ }^{(15)}$. However, since 2014 , Chile accepted and included the WHO/IDF cut-off points recommendations, based on Asian data, to prevent $\mathrm{CVD}^{(16)}$. However, it remains uncertain whether the Asian-based cut-off points are valid and represent the current anthropometric characteristics of the Chilean population.

Although some attempts have been performed to identify sex-specific cut-off points for WC in Chile, these efforts have been focused on the effect of these thresholds on overweight or cardiometabolic risk rather than MetS, a syndrome associated with a higher risk of CVD and mortality ${ }^{(17)}$. Therefore, by using nationally representative data from the three national health surveys, this study aimed to identify sex-specific cut-off points for WC in the definition of MetS for the Chilean adult population.

\section{Methods}

This study was based on participants aged $\geq 15$ years from the three available Chilean National Health Surveys conducted in 2003, 2009-2010 and 2016-2017. The Chilean National Health Surveys are large, nationally representative population-based studies of biological and lifestyle risk factors, dietary status and health conducted every 6 years in both urban and rural zones ${ }^{(18-20)}$. Data for each survey were collected by trained staff where participants were administered questionnaires, and anthropometrical and physiological measures, as well as biological samples, were obtained. A total of 15145 participants (59.6\% women) completed at least one of the three surveys (2003: $n$ 3619; 2009-2010: $n 5293$ and 2016-2017: $n$ 6233). Of these, 8182 ( $60 \%$ of women) had available information for WC and MetS and were, therefore, included in this study. More information about each survey can be found elsewhere ${ }^{(18-20)}$.

\section{Metabolic syndrome}

WC was measured at the mid-axillary line at the midpoint between the costal margin and the iliac crest by an ergonomic circumference measuring tape. Blood samples (TAG, HDL-cholesterol and glucose) were obtained by trained nurses after 8-12 h of fasting using standardised protocols. Systolic and diastolic blood pressure were measured by trained staff and derived from the mean of three readings recorded after 15 min rest ${ }^{(18-20)}$. The average value of these measures by sex is available in online Supplementary Table S2. Each MetS criterion was classified as either present or absent, following the subsequent recommendations of cut-off points: TAG $\geq 1.7 \mathrm{mmol} / \mathrm{l}$; HDLcholesterol: $<1.3 \mathrm{mmol} / 1$ in women and $<1.0 \mathrm{mmol} / 1$ in men; systolic blood pressure $\geq 130 \mathrm{mmHg}$ and/or diastolic blood pressure $\geq 85 \mathrm{mmHg}$; and fasting glucose $\geq 5.6$ $\mathrm{mmol} / \mathrm{l}$ or current treatment for diabetes ${ }^{(7-9)}$.

MetS is usually defined as the presence of at least three out of five components ${ }^{(7-9)}$. However, as WC was the outcome in the current study, instead of using three out of five in the definition of MetS, at least two out of four were used to indicate its presence following previous approaches in Latin American studies ${ }^{(21,22)}$.

\section{Statistical analyses}

All analyses were performed in Matlab R2019a. To identify WC cut-off points for the Chilean population, the original data set was divided into two different subsets employing linear binary classification. The overall predicting ability of WC on MetS was quantified by the receiver operating characteristics (ROC) curve and the area under curve (AUC). Identification of errors and correct predictions were carried out by true positives (TP), false positives (FP), false negatives $(\mathrm{FN})$, and true negatives (TN) as follows:

- Sensitivity (or true positive rate) defined as: Sensitivity $=\frac{T P}{T P+F N}$;

- Specificity (or true negative rate) defined as: Specificity $=\frac{T N}{T N+F P}$

- Type I error (or false positive rate) defined as: $R_{F P}=\frac{F P}{T N+F P}=1-$ specificity;

- Type II error (or false negative rate) defined as: $R_{F N}=\frac{F N}{T P+F N}=1-$ sensitivity; 
(a)

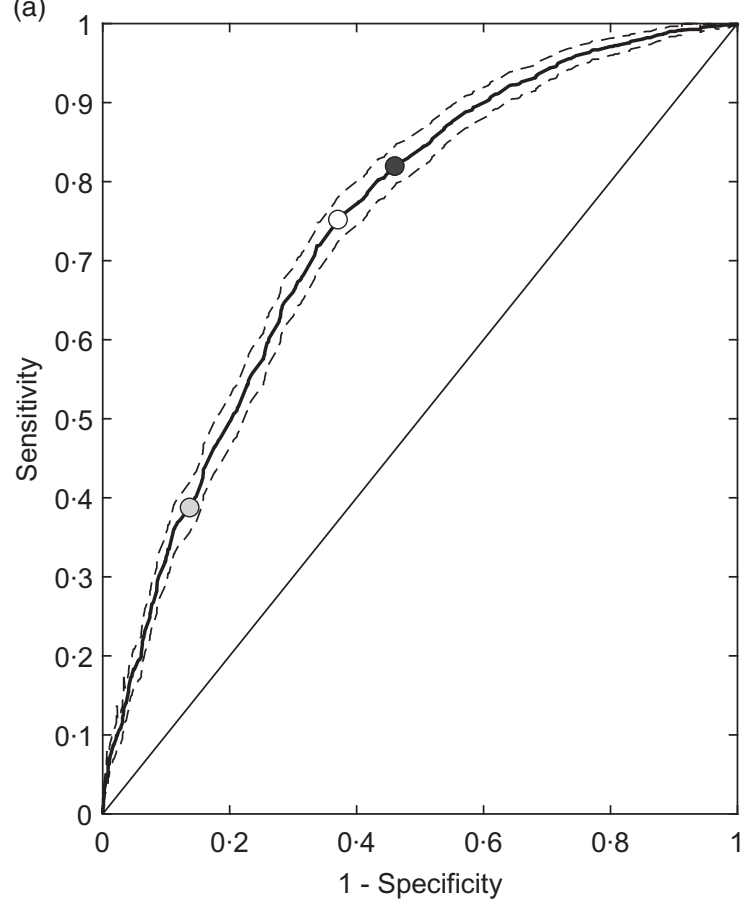

(b)

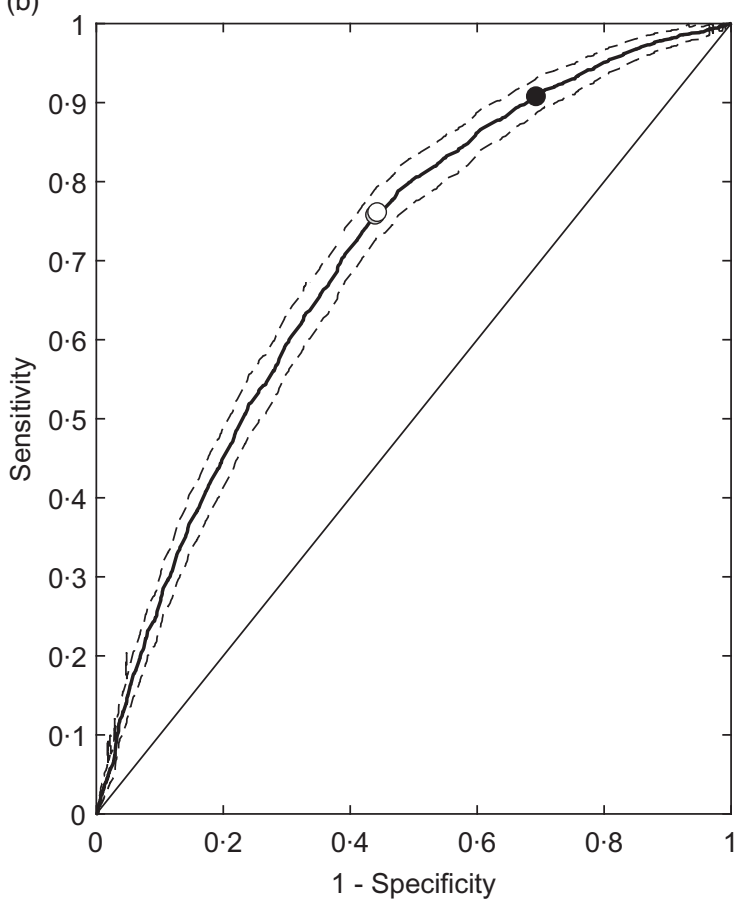

Fig. 1 Receiver operating characteristics (ROC) analyses for waist circumference in the definition of metabolic syndrome (MetS) by sex. (a) Optimal cut-off point for men in Chile, its AUC with its $95 \% \mathrm{Cl}$. In addition, the figure compares the threshold with the international recommendations. (b) Same information for women. MetS was defined as the presence of $\geq 2$ of the following conditions: TAG $\geq 1.7 \mathrm{mmol} /$; HDL-cholesterol: $<1.3 \mathrm{mmol} / \mathrm{l}$ in women and $<1.0 \mathrm{mmol} / \mathrm{l}$ in men; systolic blood pressure $\geq 130 \mathrm{~mm} \mathrm{Hg}$ and/or diastolic blood pressure $\geq 85 \mathrm{~mm} \mathrm{Hg}$; fasting glucose $\geq 5.6 \mathrm{mmol} / \mathrm{l}$ or current treatment for diabetes. (a),$- \mathrm{ROC}(\mathrm{AUC}=0.74) ;-$, Random ROC (AUC = 0.50); - -, $95 \% \mathrm{Cl} ; \bullet$, WHO/International Diabetes Federation (IDF) $=90 \cdot 0 \mathrm{~cm} ; O$, National Cholesterol Education Program Adult Treatment Panel III (ATP III) $=102.0 \mathrm{~cm}$; O, Chile $=92.3 \mathrm{~cm}$. (b) - , ROC $($ AUC $=0.71) ;-$, Random ROC $(A \cup C=0.50) ;--, 95 \% \mathrm{Cl} ; \bullet, W H O / I D F=80.0 \mathrm{~cm} ;$ O, ATP III $=88.0 \mathrm{~cm} ; 0$, Chile $=87.6 \mathrm{~cm}$

The optimal WC value was assigned as the one that minimises the errors, that is, the sum of false positive rate and the false negative rate $\left(R_{F P}+R_{F N}\right)$. We decided to use this approach, rather than other methods (e.g. Youden index, $\mathrm{LR}+, \mathrm{LR}-$ ), because we think it is important to balance between the numbers of false positive and false negative. The optimal cut-off point for the Chilean population was computed by sex considering the full data set (8182 participants). Besides, a sensitivity analysis was performed to identify if the optimal cut-off points differ by age (below and above 60 years).

Additionally, we performed 10000 bootstrapping to derive the $95 \%$ CI of cut-off points for WC, as well as the descriptive statistic. Each bootstrapping was restricted to have at least between 40 and $60 \%$ of the original population (i.e. 2000 participants were included in each bootstrapping). To compare the cut-off points derived in our study with those suggested by WHO/IDF and ATP III, we plotted the sensitivity/specificity of each of these guidelines using the Chilean data set.

Finally, the prevalence of MetS was computed depending on the cut-off points of WC. Three different points were considered for posterior analysis recovered with the presented methodology and the international recommendations. Differences between those thresholds' values were estimated.

\section{Results}

Figure 1 presents the receiver operating characteristics curve derived for the Chilean population, with their corresponding $95 \%$ CI (AUC for men 0.74 (95\% CI 0.72, 0.76); AUC for women 0.71 (95\% CI $0 \cdot 68,0 \cdot 73)$ ). The optimal cutoff points for WC, in the definition of MetS by sex, were $92.3 \mathrm{~cm} \mathrm{(95 \%} \mathrm{CI} 90.5,94.4)$ and $87.6 \mathrm{~cm} \quad(95 \% \mathrm{CI}$ $85 \cdot 8,92 \cdot 1$ ) for men and women, respectively (Fig. 1 and online Supplementary Fig. S1). These derived Chilean WC cut-off points had a higher sensitivity than the cut-off points recommended by the ATP III and higher specificity in contrast to those recommended by WHO/IDF, both in men and women. When the analyses were stratified by age (below or above 60 years), the WC cut-off points for both age groups lied within the $95 \%$ CI already derived for the whole population for both age groups (online Supplementary Fig. S2).

The descriptive statistic of the bootstrapping analyses is presented in online Supplementary Fig. S1 (median and 
(a)

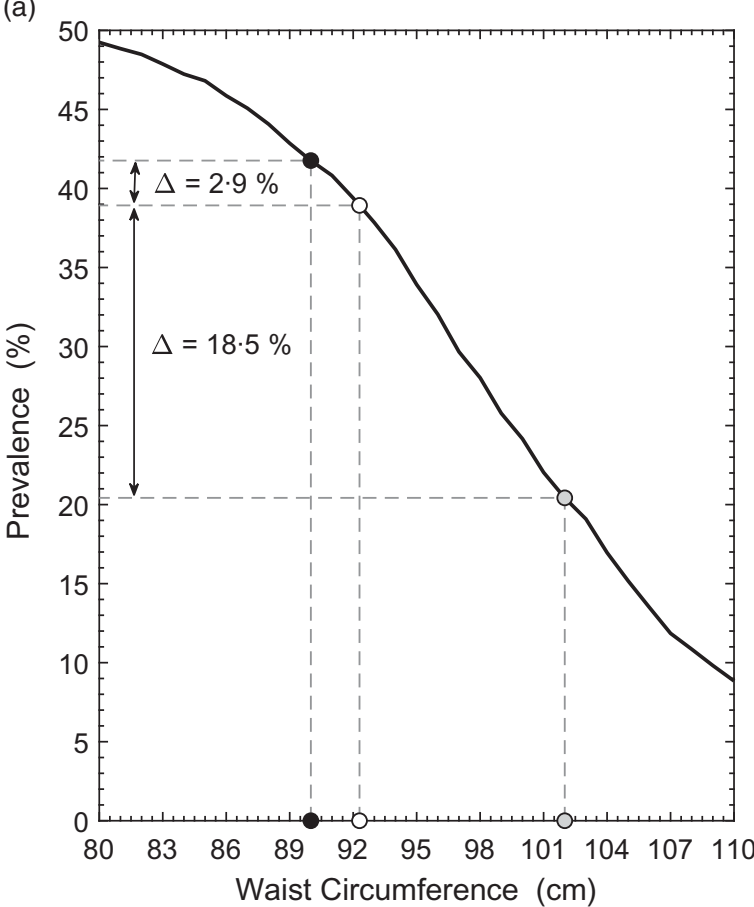

(b)

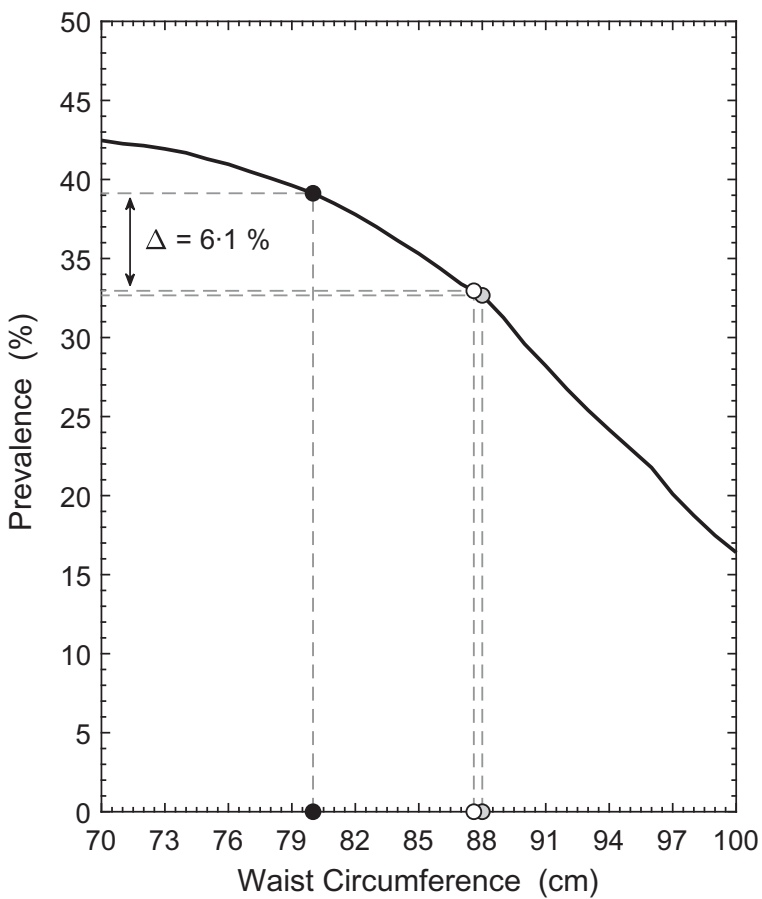

Fig. 2 Prevalence of metabolic syndrome (MetS) in Chilean adults using different cut-off points for waist circumference (WC). (a) Prevalence of MetS in men using different thresholds of WC. In addition, this figure shows the delta between the threshold identified and the international recommendations. (b) Same information for women. For this Figure, MetS was defined as the presence of $\geq 2$ of the following conditions: TAG $\geq 1.7 \mathrm{mmo} / / / \mathrm{HDL}$-cholesterol: $<1.3 \mathrm{mmol} / / \mathrm{in}$ women and $<1.0 \mathrm{mmo} / / \mathrm{in} \mathrm{men}$; systolic blood pressure $\geq 130 \mathrm{~mm} \mathrm{Hg}$ and/or diastolic blood pressure $\geq 85 \mathrm{~mm} \mathrm{Hg}$; fasting glucose $\geq 5.6 \mathrm{mmol} / /$ or current treatment for diabetes plus different cut-off points of WC. (a) —, Prevalence of MetS; - - , WHO/International Diabetes Federation (IDF) $=90.0 \mathrm{~cm}$; -0-, National Cholesterol Education Program Adult Treatment Panel III (ATP III) $=102.0 \mathrm{~cm}$; - - , Chile $=92.3 \mathrm{~cm}$. (b) —, Prevalence of MetS; - -, WHO/IDF = $80.0 \mathrm{~cm} ;-0-$, ATP III $=88.0 \mathrm{~cm} ;-0-$, Chile $=87.6 \mathrm{~cm}$

mode). The probability of having a cut-off point according to the WHO/IDF was 6.6 and $0.0 \%$ for men and women, respectively. In turn, according to ATP III, it was 0.0 and $62.2 \%$ for men and women, respectively. Therefore, the ATP III recommendation for Chilean men and the WHO recommendation for Chilean women can be considered outliers (online Supplementary Fig. S1).

Finally, the prevalence of MetS using different cut-off points for WC is presented in Fig. 2. Using the identified cut-off point of $92.3 \mathrm{~cm}$ in men, the prevalence of MetS was $38.9 \%$. This prevalence was $2.9 \%$ lower and $18.5 \%$ higher than the WHO/IDF and ATP III recommendations, respectively (Fig. 2(a)). For women, the identified WC cutoff point of $87.6 \mathrm{~cm}$ was associated with a $33 \%$ prevalence of MetS, which was $6.1 \%$ lower and only $0.4 \%$ higher than the WHO/IDF and ATP III recommendations, respectively (Fig. 2(b)).

\section{Discussion}

Since 2014, Chile has used the WHO/IDF recommendation for WC cut-off points for the Asian populations ${ }^{(16)}$. This recommendation was established in 2005 due to the lack of information for South and Central American populations and, after 15 years, still prevails ${ }^{(14)}$. To our knowledge, this is the first study identifying sex-specific cut-off points for WC in the definition of MetS for the Chilean population. The main findings of this study provide evidence that the current suggestions of using WC cut-off points derived for Asian populations may not be appropriate for the Chilean population, especially for women who - based on our estimates - should have a cut-off point $7.6 \mathrm{~cm}$ higher than the current recommendation. In our study, the cut-off points derived for women were closer to those suggested by the ATP III recommendation for American women $(88.0 \mathrm{~cm})$. However, for men, we found that the WC cutoff $(92.3 \mathrm{~cm})$ was somehow in the middle between the Asian $(90.0 \mathrm{~cm})$ and the IDF recommendations for Caucasian men $(94.0 \mathrm{~cm})^{(3,14)}$. In addition, using the identified cut-off points, the prevalence of MetS was 2.9 and $6.1 \%$ lower than the current recommendation for men and women, respectively. In this regard, although our cut-off points were closer to those recommended by the WHO/IDF for men, adopting the WHO/IDF recommendation for women could overestimate the number of individuals at risk. Changing these cut-off points for women may decrease the prevalence of this syndrome in Chile. Therefore, considering that the prevalence of MetS is highly sensitive when different thresholds for WC are used, more 
conservative cut-off points (i.e. low values) could not be necessarily representative of the national reality. The aforementioned motivates its calibration for each population and a more in-depth study across countries.

BMI and WC are both measures of adiposity. However, the cut-off points for overweight and obesity are different in Chile and Asia (BMI $\geq 25$ and $\geq 23 \mathrm{~kg} / \mathrm{m}^{2}$, respectively) ${ }^{(23)}$. Therefore, considering the same cut-off points for WC for both populations could not be justified. Moreover, the Asian population in Chile represents only $1.2 \%$ of the total Chilean population. In consequence, there are no foundations in the use of these thresholds due to the underlying differences in anthropometry and a small proportion in the population ${ }^{(24)}$.

Although this is the first study in Chile that suggests sexspecific cut-off points for WC in the definition of MetS, a previous study, conducted by Villanueva et al. $^{(17)}$, suggested sex-specific cut-off points for abdominal obesity to identify cardiovascular risk factors in adults. The authors demonstrated that thresholds of 93 and $88 \mathrm{~cm}$ in non-obese men and women, and 91 and $83 \mathrm{~cm}$ in overweight/obese men and women were associated with cardiovascular risk factors; that is, our findings were similar to those found for Villanueva et $a{ }^{(17)}$ for non-obese adults. Nevertheless, It is important to highlight that the Villanueva study and our study are different in the number of participants included and the identification of cut-off points in both studies had a different aim.

Other South American studies have also found similar results using similar approaches. Aschner et al. ${ }^{(25)}$, using data from 179 men and 278 women from Mexico, El Salvador, Venezuela, Colombia and Paraguay, identified that the optimal WC thresholds were $94.0 \mathrm{~cm}$ and between 90.0 and $92.0 \mathrm{~cm}$ for South American men and women, respectively ${ }^{(25)}$. This recommendation has also been considered by the Latin American Association of Diabetes. In Brazil, after analysing 6772 men and 8121 women, Cardinal et $a l .{ }^{(21)}$ found cut-off points of 92.0 and $86.6 \mathrm{~cm}$ for men and women, respectively ${ }^{(21)}$. In Colombia, MoraGarcía identified a threshold of $85.0 \mathrm{~cm}$ in women ${ }^{(22)}$. In Venezuela, Bermúdez et al. ${ }^{(26)}$ found that 95.2 and $90.2 \mathrm{~cm}$ were the cut-off points with the highest sensitivity after analysing 1902 adults from Maracaibo. Therefore, all the reported South American cut-off points (including those of this study) are far from the WHO/IDF recommendations for South Americans. Furthermore, as sex-specific cut-off points to determine central obesity should not be applied universally among different ethnicities or countries, future work should aim to define ethnic and country-specific cut-off points in other populations.

\section{Strength and limitations}

This study was performed employing all the available data from the Chilean National Health Surveys that are a nationally representative sample of the Chilean adult population.
In addition, all blood samples (TAG, HDL-cholesterol and glucose), systolic blood pressure and diastolic blood pressure measures used to define MetS were obtained by trained nurses using standardised protocols ${ }^{(18-20)}$. However, these data were obtained during three different periods and on different participants. Further investigations considering a larger data set should be carried out to generalise the obtained results.

\section{Conclusion}

In conclusion, the current WHO/IDF recommendation for WC was not supported by Chilean data for women, but it was closer for men. Using the three Chilean National Health Surveys, we identified that 92.3 and $87.6 \mathrm{~cm}$ could be better cut-off points for WC in the definition of MetS. As a result, the current ATP III recommendation for American women $(88.0 \mathrm{~cm})$ and the IDF for Caucasian men $(94.0 \mathrm{~cm})$ are closer to the Chilean reality. Future prospective studies should corroborate this information investigating the association of these cut-off points with incidence and mortality for CVD and all-cause mortality.

\section{Acknowledgements}

Acknowledgements: We thank all participants for their cooperation and the Chilean Health Ministry and School of Public Health, The Pontificia Universidad Católica de Chile for commissioning, designing and conducting the three National Health Surveys. Financial support This study was funded by the Chilean Health Ministry as part of the health surveillance in Chile. The funders of the study had no role in study design, data collection, data analysis, data interpretation or any decision related to this article. Conflict of interest: None to declare. Authorship: F.P.-R., C.C.-M. and A.P. generated the research question. F.P.-R. and A.P. planned the analysis. F.P.-R. performed the literature search. F.P.-R. and A.P. performed the analyses with support from C.C.-M. F.P.-R. and A.P. wrote the first draft of the manuscript. All authors critically reviewed this and previous drafts. All authors approved the final draft for submission. F.P.R. is the guarantor. Ethics of buman subject participation: The Chilean National Health Surveys were funded by the Chilean Ministry of Health and led by the Department of Public Health of the Pontificia Universidad Católica de Chile. The Chilean National Health Surveys were approved by the Ethics Research Committee of the Faculty of Medicine at the same university. All participants who participated provided written informed consent.

\section{Supplementary material}

For supplementary material accompanying this paper visit https://doi.org/10.1017/S1368980020001469 


\section{References}

1. Hotez PJ \& Peiperl L (2015) Noncommunicable diseases: a globalization of disparity? PLoS Med 12, e1001859e1001859.

2. Magnusson RS (2010) Global health governance and the challenge of chronic, non-communicable disease. J Law Med Ethics 38, 490-507.

3. Alberti KG, Eckel RH \& Grundy SM et al. (2009) Harmonizing the metabolic syndrome: a joint interim statement of the International Diabetes Federation Task Force on Epidemiology and Prevention; National Heart, Lung, and Blood Institute; American Heart Association; World Heart Federation; International Atherosclerosis Society; and International Association for the Study of Obesity. Circulation 120, 1640-1645.

4. Chatterjee A, Harris SB \& Leiter LA et al. (2012) Managing cardiometabolic risk in primary care: summary of the 2011 consensus statement. Can Fam Physician 58, 389-e201.

5. Reaven GM (1988) Role of insulin resistance in human disease. Diabetes 37, 1595.

6. Alberti KG \& Zimmet PZ (1998) Definition, diagnosis and classification of diabetes mellitus and its complications. Part 1: diagnosis and classification of diabetes mellitus provisional report of a WHO consultation. Diabet Med 15, 539-553.

7. Huang PL (2009) A comprehensive definition for metabolic syndrome. Dis Model Mech 2, 231-237.

8. Saklayen MG (2018) The global epidemic of the metabolic syndrome. Curr Hypertens Rep 20, 12-12.

9. Kassi E, Pervanidou P \& Kaltsas G et al. (2011) Metabolic syndrome: definitions and controversies. BMC Med 9, 48-48.

10. Misra A, Chowbey P \& Makkar BM et al. (2009) Consensus statement for diagnosis of obesity, abdominal obesity and the metabolic syndrome for Asian Indians and recommendations for physical activity, medical and surgical management. J Assoc Physicians India 57, 163-170.

11. Kim CH, Kim HK \& Kim EH et al. (2012) Impact of body mass index on the predictive ability of body fat distribution for type 2 diabetes risk in Koreans. Diabetic Medicine 29, 1395-1398.

12. Resnick HE, Valsania P \& Halter JB et al. (1998) Differential effects of BMI on diabetes risk among black and white Americans. Diabetes Care 21, 1828-1835.

13. Stommel M \& Schoenborn CA (2010) Variations in BMI and prevalence of health risks in diverse racial and ethnic populations. Obesity 18, 1821-1826.

14. Alberti KG, Zimmet P \& Shaw J (2005) The metabolic syndrome - a new worldwide definition. Lancet $\mathbf{3 6 6}$, 1059-1062.

15. National Cholesterol Education Program (NCEP) Expert Panel on Detection E, and Treatment of High Blood Cholesterol in Adults (Adult Treatment Panel III) (2002)
Third report of the National Cholesterol Education Program (NCEP) expert panel on detection, evaluation, and treatment of high blood cholesterol in adults (adult treatment panel III) final report. Circulation 106, 3143-3421.

16. MINSAL (2014) ENFOQUE DE RIESGO PARA LA PREVENCIÓN DE ENFERMEDADES CARDIOVASCULARES. Ministerio de Salud, Gobierno de Chile. http://www. repositoriodigital.minsal.cl/handle/2015/907 (accessed October 2019).

17. Villanueva B, Arteaga A \& Maiz A et al. (2018) Abdominal obesity is a common finding in normal and overweight subjects of Chile and is associated with increased frequency of cardiometabolic risk factors. PLOS ONE 13, e0194644.

18. MINSAL (2010) Encuesta Nacional de Salud 2009-2010, Chile. Ministerio de Salu, Gobierno de Chile. https://www. minsal.cl/portal/url/item/bcb03d7bc28b64dfe0400101650 12d23.pdf (accessed August 2019).

19. MINSAL (2003) Encuesta Nacional de Salud 2003, Chile. Ministerio de Salud, Gobierno de Chile. http://www. medicinadefamiliares.cl/Protocolos/encnacsalres.pdf (accessed August 2019).

20. MINSAL (2017) Encuesta Nacional de Salud 2016-2017, Chile. Ministerio de Salud, Gobierno de Cbile. https:// www.minsal.cl/wp-content/uploads/2017/11/ENS-2016-17_ PRIMEROS-RESULTADOS.pdf (accessed August 2019).

21. Cardinal TR, Vigo A, Duncan BB et al. (2018) Optimal cut-off points for waist circumference in the definition of metabolic syndrome in Brazilian adults: baseline analyses of the Longitudinal Study of Adult Health (ELSA-Brasil). Diabetol Metab Syndr 10, 49.

22. Mora-García GJ, Gómez-Camargo D, Mazenett E et al. (2014) Anthropometric parameters' cut-off points and predictive value for metabolic syndrome in women from Cartagena, Colombia. Salud Publica Mex 56(2): 146-153.

23. WHO (2004) Appropriate body-mass index for Asian populations and its implications for policy and intervention strategies. Lancet 363, 157-163.

24. INE (2018) Características Sociodemográficas de la Inmigración Internacional en Chile. Instituto Nacional de Estadística, Gobierno de Chile. http://www.censo2017.cl/ descargas/inmigracion/181123-documento-migracion.pdf (accessed October 2019).

25. Aschner P, Buendia R, Brajkovich I et al. (2011) Determination of the cutoff point for waist circumference that establishes the presence of abdominal obesity in Latin American men and women. Diabetes Res Clin Pract 93 , 243-247.

26. Bermúdez JV, Rojas J, Salazar J et al. (2014) Optimal waist circumference cut-off point for multiple risk factor aggregation: results from the Maracaibo City Metabolic Syndrome Prevalence Study. Epidemiol Res Int 2014, 9. 